\title{
Establishing a Framework for the Use of Social Media in Pharmacovigilance in Europe
}

\author{
Sabine Brosch ${ }^{1} \cdot$ Anne-Marie de Ferran ${ }^{2} \cdot$ Victoria Newbould $^{1} \cdot$ Diane Farkas $^{2} \cdot$ Marina Lengsavath $^{2} \cdot$ Phil Tregunno $^{3}$
}

Published online: 30 March 2019

(c) The Author(s) 2019

\begin{abstract}
The Innovative Medicines Initiative (IMI) WEB-RADR (Web-Recognising Adverse Drug Reactions) project looked at opportunities and challenges in using social media in pharmacovigilance as a rapidly evolving source of large, real-time data, which could provide new information on the actual use of medicines and potential safety issues. Two of the objectives were to develop principles for continuous monitoring of the safety of medicines without overburdening established pharmacovigilance systems and to propose a regulatory framework on the use of social media in pharmacovigilance. As a starting point, a review of existing legal requirements and regulatory guidance on social media use in pharmacovigilance was performed based on a survey conducted in 2014-2015. Furthermore, input from two large stakeholder workshops and evidence gathered from the research performed by WEB-RADR on the analysis of social media data were taken into consideration. Whilst analytical results of WEB-RADR indicated limited value of social media in detecting or confirming signals for a majority of the drugs studied, it is important to establish a regulatory framework for the use of social media in pharmacovigilance. Thus, the screening and reporting of suspected adverse reactions remains an important pillar in monitoring the safety and efficacy of medicines and the identification of new safety issues. Principles as to how social media can be used in pharmacovigilance are absolutely needed to provide clarity to patients, healthcare professionals, medicines regulators and the pharmaceutical industry.
\end{abstract}

The views expressed in this article are the personal views of the authors as a result of the IMI WEB-RADR project and may not be understood or quoted as being made on behalf of or reflecting the position of the agencies or organisations with which the authors are affiliated.

Electronic supplementary material The online version of this article (https://doi.org/10.1007/s40264-019-00811-8) contains supplementary material, which is available to authorized users.

Sabine Brosch

sabine.brosch@ema.europa.eu

1 Surveillance and Epidemiology Services, Pharmacovigilance and Epidemiology Department, European Medicines Agency (EMA), 30 Churchill Place, Canary Wharf, London E14 5EU, UK

2 Sanofi, 1, Avenue Pierre Brossolette, 91385 Chilly-Mazarin Cedex, France

3 Medicines and Healthcare products Regulatory Agency, 10 South Colonnade, Canary Wharf, London E14 4PU, UK

\section{Key Points}

Social media can offer opportunities in pharmacovigilance by providing interactive platforms as well as access to large and real-time datasets, although it should be acknowledged that WEB-RADR (Web-Recognising Adverse Drug Reactions) results indicate limited value in detecting signals.

A key challenge is obtaining clarity on the regulatory requirements that arise in using social media such as the processing and reporting of safety information.

Principles for the use of social media in pharmacovigilance are put forward that take into account existing legal requirements and the need to provide continuous monitoring of the safety of medicines. 


\section{Introduction}

New tools and data sources such as social media provide a variety of public health opportunities, including in the area of drug safety and pharmacovigilance, a dynamic clinical and scientific discipline [1]. Pharmacovigilance is defined by the World Health Organization (WHO) as "the science and activities relating to the detection, assessment, understanding and prevention of adverse effects or any other drug-related problem" [2].

In the European Union (EU) pharmacovigilance requirements are set out in Directive 2001/83/EC [3], Regulation (EC) 726/2004 [4] and the Commission Implementing Regulation (EU) 520/2012 [5]. The legislation is supplemented by the Good Pharmacovigilance Practices (GVP) applicable to marketing authorisation holders (MAHs), the European Medicines Agency (EMA) and medicines regulatory authorities in EU Member States [6]. GVP Module VI refers to digital media as "web sites, web pages, blogs, vlogs, social networks, internet forums, chat rooms and health portals" [7] and outlines the screening and adverse drug reaction (ADR) reporting obligations of MAHs depending whether the digital media are company-sponsored or not. A digital medium is considered to be company sponsored if it is owned, paid for and/ or controlled by the MAH. The frequency of the screening should allow for valid individual case safety reports (ICSRs) to be submitted to the competent authorities within the appropriate regulatory submission timeframes based on the date the information was posted on the internet site/digital medium. However, if an MAH becomes aware of an ADR on website media that it does not manage, the MAH should nevertheless review the case and determine whether it should be reported [7]. Unsolicited reports of suspected ADRs collected from digital media are reportable as spontaneous reports based on the criteria for a valid ICSR, which should include the following minimum criteria: at least one identifiable reporter, one single identifiable patient, at least one suspect adverse reaction and at least one suspect medicinal product $[7,8]$. For cases from digital media, the identifiability of the reporter refers to the possibility of verification of the existence of a real person based on the information available, e.g. an email address with a valid format has been provided. If the country of the primary source is missing, the country where the information was received, or where the review took place, should be used as the primary source country [7].

In a broader context, MAHs need to routinely re-evaluate the risk-benefit balance of their own medicinal products in exposed populations, with a structured evaluation to be undertaken. This refers to a comprehensive, concise and critical analysis of the risk-benefit balance of the medicinal product, taking into account new or emerging information on risks and benefits at defined timepoints in the lifecycle of a medicinal product. This is performed in periodic safety update reports (PSURs) as set out in GVP Module VII [9] as well as ongoing pharmacovigilance and risk management (GVP Module V) activities to facilitate optimisation of the risk-benefit balance through effective risk minimisation measures $[10,11]$.

GVP Module IX sets out the process for signal management and defines sources of data and information as diverse and potentially including "all scientific information concerning the use of medicinal products and the outcome of the use, i.e. quality, non-clinical and clinical data (including pharmacovigilance and pharmacoepidemiological data)" [12]. Finally, GVP Module VIII sets out the principles for post-authorisation safety studies (PASS) relating to authorised medicinal products conducted with the aim of identifying, characterising or quantifying a safety hazard, confirming the safety profile of the medicinal product, or of measuring the effectiveness of risk management measures [13]. Here, social media lends itself to facilitate the performance of such studies.

The use of social media and associated regulatory challenges are becoming widely discussed. Whilst some experts believe that monitoring of the safety of medicines via social media could prove to be an efficient and expeditious means of post-market safety surveillance overcoming limitations of traditional ADR reporting systems [14], others stress that ADR reporting methodologies could add social media as a source of information for the benefit-risk evaluation of medicines [15].

The EU Innovative Medicines Initiative (IMI) WEBRADR (Web-Recognising Adverse Drug Reactions) [16] consortium therefore set itself the task of assessing the use of social media in safety monitoring [17]. The project was organised in several work packages (WPs), of which two focused on collecting and analysing social media data to understand the value in identifying new safety signals (WP $2 \mathrm{~A}$ and 2B). The objective of WP1 was to put forward regulatory recommendations that can inform the future development or updating of pharmacovigilance guidance such as the GVP modules to address identified challenges. These relate, for example, to large numbers of ADRs resulting from social media data that are to be processed. Ramble and Balatero [18] suggest that "Pharmaceutical companies fear that opening up social listening around medicinal products by MAHs will expose them to posts where authors share an undesirable experience associated with the use of a medical product in a patient, and that MAHs will need to invest heavily in resources to manage adverse event reporting" [18]. This is further acknowledging that these data are derived outside the classic spontaneous reporting systems where the reporting is dependent on initiative and motivation of the reporters [19], 
and that social media data richness may be variable and the follow-up challenging. Taking into account that one important function of spontaneous reporting systems is the early identification of signals [20], there are generally concerns that processing social media data into pharmacovigilance databases will reduce the effectiveness of existing signal detection activities due to data dilution. One theory is that data from social media platforms could serve as an early signal from a broader patient population and could supplement conventional pharmacovigilance data sources. Another theory is that social media have limitations due to important differences in the types of information and adverse events described, inherent issues with data reliability [21] and the potential risk of diluting important signals originating from current spontaneous reporting systems [22]. Another hurdle is that the data source cannot be clearly identified for reporting as ICSRs. The International Council for Harmonisation of Technical Requirements for Pharmaceuticals for Human Use (ICH) [23] developed the E2B(R3) ICSR implementation guide [24], which allows for a report type categorisation and capturing of literature references. However, there is currently no mechanism for specifying the data source in a structured manner, e.g. the social networking site(s) [SNSs] from where the data are sourced. Therefore, this does not permit targeted and segregated signal detection and analysis of adverse reactions originating from social media in pharmacovigilance systems such as EudraVigilance [25] or easy identification of duplicated reports. Finally, lacking the classical setting of a dedicated reporter with the primary objective of reporting a suspected adverse reaction related to a medicine, ADR reporting from social media may be difficult as the four minimum criteria for a valid report [21] may not be met, e.g. in the context of social listening where the patient and medicine use are contextualised and aggregated social media datasets are obtained from social network providers. Whilst the GVP Modules in the EU provide certain guidance on the use of social media as outlined, these challenges indicate that further work in this area is required.

\section{Methods}

In order to develop principles that can address the identified challenges, WP1 members of the WEB-RADR project used three types of data sources. Firstly, drug regulatory requirements in 182 countries/jurisdictions within and outside the EU, which were reviewed to gather information on existing guidance and legal requirements on social media and drug safety. The results were published in 2016, providing a baseline understanding of current requirements and gaps requiring further clarification [26]. Secondly, the EMA organised two large workshops with members of its Healthcare Professionals and Patients' and Consumers' Working Parties, the
Pharmacovigilance Risk Assessment Committee (PRAC), representatives from Young People (Paediatric Committee), and medical ethics and data protection experts to discuss guiding principles as to how social media could facilitate the monitoring of the safety of medicines [27, 28]. Along with the outputs from these workshops, regular meetings of WP1 members focused on addressing key regulatory questions raised as part of the expert and working party interactions, of which six key topics emerged:

- What is the obligation of MAHs to screen social media for collecting ADRs?

- What constitutes a valid ADR report from social media?

- Should MAHs attempt to follow-up with social media users for the purpose of ADR reporting?

- Do MAHs need to screen social media for the purpose of signal detection?

- Does the use of social media make a difference in pharmacovigilance obligations if evidence suggests it contributes to signal detection or safety information?

- What data protection and confidentiality aspects apply to the use of public social media data?

Thirdly, scientific evidence from the WEB-RADR social media analytics and evaluation work streams [29-32] was taken into account. Considering the outputs of all three sources, key principles for a regulatory framework were developed based on the currently applicable EU legislation and ICH guidelines. Finally, PRAC was consulted resulting in the key principles outlined in Sect. 3.

\section{Results}

The framework put forward for the use of social media in pharmacovigilance focuses on two areas of the use of social media based on guidance issued by the Social Media Research Group [33]: (i) a tool to engage and interact with patient and healthcare communities; and (ii) an additional data source to research content without engaging with the researched communities. In this context social media is "a group of Internetbased applications that build on the ideological and technological foundations of Web 2.0 that allow the creation and exchange of user-generated content based on mobile and webbased technologies to create highly interactive platforms via which individuals and communities share, co-create, discuss, and modify user-generated content" [34].

\subsection{Key Principles for Use of Social Media as a Tool to Interact with Patients and Healthcare Communities}

In instances where social media are used merely as a support tool to facilitate an interactive, two-way communication 
and information exchange, the reporting obligations based on GVP modules [6] should be followed accordingly. This applies, for example, to the reporting of suspected adverse reactions in accordance with GVP Module VI [7], the review of cumulative information on risks and benefits as part of the PSUR in accordance with GVP Module VII [9], and ongoing pharmacovigilance and risk management activities set out in GVP Module V [11]. The inclusion of a definition, in particular in relation to social media either in GVP Annex I [35] or GVP Module VI [7], should be considered as part of future updates.

The use of a 'social media handle'-a username that is public and can be used to identify people online-should be considered acceptable as a valid patient or reporter identifier. In the absence of a dedicated data element in the ICH E2B ICSR format [24], the social media handle could be reflected in the data element dedicated to the reporter or patient name (if no other patient or reporter parameters for a valid case are available), a point that could be further addressed by the ICH E2B Expert Working Group.

For the processing of ADRs, the date the information was posted on a site and the date that anyone from the organisation or working on behalf of the organisation first becomes aware of the information should be captured [36]. This latter date determines the day zero for reporting, as set out in GVP Module VI [7].

Non-interactive communications, e.g. as part of risk minimisation activities, have increased patient awareness of drug safety issues [37]. In the context of such communication campaigns, a 'contact us' link is often provided on company-sponsored sites, and the MAH should remain responsible for monitoring any safety-related information received there [36].

\subsection{Key Principles for Use of Social Media Data in Pharmacovigilance}

Research or analysis of data sourced from social media without planned engagement with the explored social media community should be considered as secondary use of data, i.e. the data were previously provided/collected for a different purpose. This takes into account that research is internetbased, the data are unsolicited and real time, and the content is generated by individuals or communities with a primary objective to share, co-create, discuss and modify information and experiences in a broader context. Here the key aspect of secondary data use is that there is no planned interaction with the social media users and the primary objective of the research of the data is focused on public health protection. The use of data generated from social media that is not sponsored by a MAH [7] should be optional for the purpose of pharmacovigilance. This is based on the evidence gained from the IMI WEB-RADR and similar projects [29-31].
If data sourced from social media are utilised for pharmacovigilance activities, the following aspects should be defined and documented before any data collection, research or analysis is initiated:

(a) Purpose of the use of the social media data, e.g. signal management and/or signal validation.

(b) Choice of the social media data, e.g. specialised health communities and forums, generic SNSs such as Facebook or Twitter, including if the data are derived from public or private sites and the reason for the choice.

(c) Type of social media content to be applied, i.e. text content or image (pictures) or both.

(d) Terms of use from the data provider to document how the data can be utilised for the defined purpose.

(e) Time period covered by the dataset, including periodicity of updates and the approach to address evolving content (posts/discussions).

(f) Social media user demographics where feasible, such as general user profile information (age group, gender), including the representation of specific populations of interest.

(g) Taking into account that representativeness of social media data is an area that is still to be further explored, an approach should be defined to mitigate against any 'skewing' [33].

(h) Generalisability and replicability of the findings of the social media data in the context of the defined purpose should be documented [38].

(i) Based on the principle of secondary use of data, there should be no planned interactions with the researched community.

(j) The adopted data management approach including data curation needs to be defined upfront; this refers to the named entity recognition (NER) problem in the information retrieval community [31], which is an active research area. Examples are methods of identifying relevant medicines taking into account phonetic spellings and usage of brand and generic names, which are factors that need to be taken into account in the overall data management approach [39]. It also includes processing informal text used online where lay language is often used to describe common symptoms or experiences rather than specific illnesses. Approaches on the application of natural language processing (NLP) tools should be defined as this will have an impact on how data are being further analysed [40].

(k) Methods and algorithms to be applied and frequency of analysis to be performed are to be documented. Given the variety and potential size of social media data, new and dynamic approaches to existing quantitative and qualitative research techniques should be considered carefully. The Social Media Research Group 
have developed a social media methodology spectrum which outlines a broad range of social media-derived data types that can help answer social research questions and proposes traditional and new research methods that could be useful analytical tools for the different data types [33].

(l) It is important that personal data protection requirements, including safeguards of data (security and access) and retention periods, are adhered to in line with the applicable data protection legislation [41].

(m) Ethical standards should be applied accordingly [42].

\subsubsection{Key Principles for Use of Social Media for the Purpose of Signal Detection or Validation}

Overall, social media such as Facebook and Twitter are currently not considered worthwhile to employ for the purpose of broad-ranging statistical signal detection at the expense of other pharmacovigilance activities. Although future improvements to ADR recognition in social media posts in terms of performance and coverage may revise this recommendation, social media is not expected to become a firstline signal detection tool. It may, however, serve as a useful complement in specific niche areas [32]. Where an organisation decides on the use of social media data for signal detection and/or signal validation, the reporting of ADRs in the form of ICSRs should not be required as set out in GVP Module VI [7]. This is based on the principle of the 'secondary use of data' explained in Sect. 3.2. Follow-up with social media users should also not be a requirement. The signal detection and validation process, handling of the results and assessment of any findings should be based on the principles set out in GVP Module IX [12] and fully documented. Information obtained through social media data that contribute to the safety profile should be included within relevant regulatory procedures [10] associated with the medicinal product, including PSURs [9] and risk management/minimisation plans [11]. In case of an emerging safety issue, this should be notified in writing to the relevant medicines regulatory authority/ies in accordance with the requirements set out in GVP Module IX [12].

\subsubsection{Key Principles for Use of Social Media as a Data Source for Non-Interventional Studies}

For the conduct of non-interventional studies based on data sourced from social media, it is recommended that the principles of 'secondary use of data' as outlined in GVP Module VI should be applied [7] when the social media are not used for primary (prospective) data collection. A protocol in accordance with GVP Module VIII [13] should be developed, which precisely describes all activities performed in the study, allowing the study to be reproduced. The protocol should be amended and updated as needed and justified [43]. EU guidance on the format and content of the protocol of non-interventional PASS [44] provides a template for protocols and may be applied to all non-interventional PASS, including meta-analyses and systematic reviews. The following should be taken into account:

(a) Reporting of ADRs in the form of ICSRs and followup with social media users should not be required in accordance with GVP Module VI [7] and the conduct of non-interventional studies based on secondary use of data [20].

(b) Reports of ADRs should be summarised as part of any interim safety analysis and in the final study report unless the protocol provides for different reporting [7].

(c) Information obtained through social media data that contribute to the safety profile of a medicinal product should be included within relevant regulatory procedures [10] associated with the product including signal management [12], PSURs [9] and risk management/ minimisation plans [11].

\subsubsection{Key Principles for Use of Social Media as a Data Source for Safety Monitoring Activities}

The primary objective of the data monitoring activities should focus on public health protection and the principles of GVP Module VI for non-interventional studies based on the secondary use of data [7]. This applies where the content and activity of social media are researched without planned engagement with the researched community, e.g. to contextualise patients and drug use.

\section{Discussion}

\subsection{Social Media as a Tool to Engage and Interact with Patient and Healthcare Communities}

Evidence from the two WEB-RADR workshops showed social media can be utilised as interactive platforms to proactively engage and interact with patient and healthcare communities, and there are numerous examples where their use has proven beneficial. Locating patients with particular diseases or symptoms can help identifying potential clinical trial candidates $[45,46]$. By listening to online conversations, companies developing or testing new medicines can track patient symptoms and also identify healthcare professionals who might support the trial [47]. This approach enables companies to develop strategies for targeting and engaging candidates with other digital initiatives, such as online clinical trials tools $[47,48]$. 
Non-interventional studies [44] involving primary data collection such as prospective observational studies and registries in which the data collected are derived from routine clinical care could also be facilitated by social media. The data can be actively collected by an organisation using interviews, questionnaires and patient follow-up as part of normal clinical practice, thus utilising modern technologies. Examples are provided in an overview of recent trends in qualitative and mixed methods of social media research designs [49].

Pharmaceutical companies may also see the opportunity to create patient support groups and communities, e.g. as part of patient support programmes (PSPs), thus providing nurse advice, tips and interviews with medical experts and facilitating connections between patients and others in the community by joining disease-related conversations such as on multiple sclerosis [50] and diabetes mellitus [51] or selfassessing disease knowledge.

The application of social media technology is also recognised as an emerging trend for patients who are seeking health information [52], and therefore can provide a modern and interactive platform to engage with patient communities. As outlined by Boucher [53], social media have the potential to improve access and quality of care and to provide patients with a new type of support.

Social media are also used in conducting market research, such as consumer healthcare studies on medicinal product formulations and flavour or consumer focus groups on advertising campaigns to choose the best advertisement. Other examples are surveys to investigate the willingness to pay for a new medicinal product or to determine reasons for switching medications or discontinuing treatments [54].

Social media can be also applied in support of risk minimisation measures such as educational programmes [55]. This includes, for example, product-specific risk management plans (RMPs), which aim to facilitate informed decision-making to support risk minimisation when prescribing, dispensing and/or using a medicinal product [56]. While routine measures [11] are applied to every medicinal product, additional risk minimisation activities are usually only introduced when they are deemed to be essential for the safe and effective use of the medicinal product. Here social media can prove to be an effective communication tool to enrol patients, prescribers, pharmacies and healthcare facilities [57], e.g. in situations when prescription is only possible as part of a restricted programme or based on the dissemination of disease-specific educational information aimed at patients to better understand their disease and to facilitate engagement with healthcare professionals to improve health outcomes [58].

Recent innovative initiatives have looked at the possibilities of using a social media application for the collection of ADR reporting and monitoring and exchange of safety information [17]. The use of social media to actively collect adverse reactions from patients and healthcare professionals can therefore be considered as a potential new mechanism to collect data in a real-time and real-life setting. A study to determine the attitude and behaviour of the pharmaceutical industry, healthcare professionals and the public towards the concept of using social media as a tool for ADR reporting and monitoring showed that $83 \%$ of the general public participants agreed that patients would be more inclined to report suspected ADRs via digital and social media if the correct measures to protect personal data were in place [14]. Sixty-three percent of the healthcare professionals believed that the concept of utilising social media for patient safety purposes would be feasible and $71 \%$ of the pharmaceutical companies stated they would consider this a possible tool from a legislative and industry perspective [14].

Taking into account these trends, the framework developed in Sect. 3.1 should facilitate the use of social media as a tool to interact and engage with the patient and healthcare community.

\subsection{Social Media as an Additional Data Source in Pharmacovigilance}

Social media in pharmacovigilance has been gaining interest, with various sources considered for detecting suspected ADRs. This includes general purpose SNSs such as Twitter or health and support networks such as PatientsLikeMe ${ }^{\circledR}$, DailyStrength and MedHelp ${ }^{\circledR}$ [31]. Social media are considered a rich and real-life-based data source, which might assist in identifying new safety issues and important insight in areas such as usage of medicines during pregnancy, offlabel use [59], misuse, medication errors or lack of efficacy related to medicinal products. In addition, there is an increasing understanding of the importance of incorporating patients' needs and concerns into care delivery to provide more patient-centred care [60]. Social media hold the potential to address this need and provide healthcare practitioners with timely access to a large pool of patients' concerns [61]. User posts in social media may contain information about treatment outcomes and provide early access to reported ADRs that could be beneficial for medicines regulators and the pharmaceutical industry. The type and volume of ADR information that social media make available are not easily obtainable by other means. This includes ADRs experienced by patients with special conditions such as rare diseases, pregnant/nursing women, elderly people or patients with co-morbidities who are usually excluded from clinical trials [62].

Taking into account the potential data richness of social media, especially in areas that are not necessarily covered through spontaneous reporting, Harpaz et al. [63] have 
highlighted the potential of social media for public health monitoring due to the availability of large amounts of data that are "internet-based, patient-generated, unsolicited, and up-to-date" [63]. As stated by Curtis et al. [64], there are many examples where social media have been studied for a variety of health conditions, including cancer $[65,66]$, diabetes [67, 68] and obesity [69].

In light of the high interest in new data sources, Sect. 3.2 provides a framework on how social media data can be used to support pharmacovigilance activities.

\subsection{Social Media for the Purpose of Signal Detection or Validation}

Social media data can be considered as one of multiple sources to identify early signals related to rare adverse reactions or other factors such as misuse, abuse, overdose, medication errors, occupational exposure and impact of medicines on the quality of life. This is in line with the definition of signal detection, which refers to "the act of looking for and/or identifying signals using data from any source" [70]. Furthermore, social media can prove to be a valuable source in validating signals [71], e.g. to confirm signals that have arisen in other reporting systems. Sarker et al. [62] highlight that while the current focus of social media analytics is to identify early safety signals, it could potentially be used to validate or reject signals that have arisen from classical ADR reporting systems [62]. The results of WEB-RADR, however, suggests that using currently available methods for ADR identification, broad-ranging statistical signal detection in Twitter and Facebook performs poorly and therefore cannot be recommended at the expense of other pharmacovigilance activities [32]. These insights have been reflected in the framework for the use of social media data in signal detection (see Sect. 3.2.1).

\subsection{Social Media as a Data Source for Non-Interventional Studies}

Social media data may also lend itself as a data source for non-interventional studies. Examples are the evaluation of patterns of the usage of medicines, including the potential for off-label use, lack of efficacy or use of medicines during pregnancy, to measure the effectiveness of risk management measures or to perform health outcome assessments. Curtis et al. [64] studied the use of data gathered from social media to complement traditional data sources to answer questions regarding comparative effectiveness and safety. To do this, they analysed publicly available social media data including Facebook, blogs and discussion boards for posts mentioning inflammatory arthritis (e.g. rheumatoid, psoriatic). Other examples are the conduct of an observational study on the associations between exposure to and expression of negative opinions about human papillomavirus vaccines on social media [72] or a pilot study performed in electronic health records and social media with the objective of better understanding differences and similarities between clinician-reported ADRs and patients' concerns regarding aspirin and atorvastatin [59]. Based on the potential of social media data to conduct non-interventional studies, specific key principles for such settings have been put forward (see Sect. 3.2.2).

\subsection{Key Principles for Use of Social Media as a Data Source for Safety Monitoring Activities}

The monitoring of social media sites to determine what the public are discussing, saying or sharing about medicinal products, diseases, conditions and treatment options is also on the rise. This can be performed prospectively or retrospectively in company- and non-company-sponsored sites. MAHs may 'listen to' user-generated content from key healthcare opinion leaders or patient group representatives for a defined period of time [36]. Other examples are patient-reported outcomes from web-based sources, which are becoming increasingly important since they provide opportunities for pharmaceutical industry and medicines regulators to understand the benefits and risks of medicines in a real-world context [15]. These trends are indicative of an increasing use of social media to support safety monitoring and for which a regulatory framework is needed (see Sect. 3.2.3).

\section{Conclusion}

The EU IMI WEB-RADR project provided a unique platform to discuss regulatory aspects of the use of social media in the area of pharmacovigilance, evaluate regulatory provisions, share experiences and learn from the evidence resulting from the project activities. This allowed the project to reflect on how social media can be effectively utilised and develop a framework that can inform the preparation/updating of regulatory guidance. The applied risk-based approach aims to allow better utilisation of these new tools and data sources. The objectives of the developed principles are to ensure a continuous monitoring of the safety of medicines and a timely identification of potential safety issues without overburdening established pharmacovigilance systems. Overall, it should be recognised that results from the analytical research conducted by the IMI WEB-RADR project clearly suggest that broadranging statistical signal detection in Twitter and Facebook performs poorly based on current available methods 
for adverse event recognition and cannot be recommended at the expense of other pharmacovigilance activities [32].

Acknowledgements The authors would like to acknowledge their WP1 colleagues, Vicky Edwards (Abbvie) and Gilles Touraille (EMA).

\section{Compliance with Ethical Standards}

Funding The research leading to these results was conducted as part of the WEB-RADR consortium (http://webradr.eu), which is a public-private partnership coordinated by the Medicines and Healthcare products Regulatory Agency in Europe. The WEB-RADR project has received support from the Innovative Medicine Initiative Joint Undertaking (http://www.imi.europa.eu) under Grant Agreement No. 115632, resources of which are composed of financial contributions from the European Union's Seventh Framework Programme (FP7/2007-2013) and European Federation of Pharmaceutical Industries and Associations companies' in-kind contributions.

Conflict of interest Sabine Brosch, Victoria Newbould, Anne-Marie de Ferran, Marina Lengsavath, Diane Farkas and Phil Tregunno have no conflicts of interests to declare.

Open Access This article is distributed under the terms of the Creative Commons Attribution-NonCommercial 4.0 International License (http://creativecommons.org/licenses/by-nc/4.0/), which permits any noncommercial use, distribution, and reproduction in any medium, provided you give appropriate credit to the original author(s) and the source, provide a link to the Creative Commons license, and indicate if changes were made.

\section{References}

1. Jeetu G, Anusha G. Pharmacovigilance: a worldwide master key for drug safety monitoring. J Young Pharm. 2010;2(3):315-20. https://doi.org/10.4103/0975-1483.66802.

2. World Health Organization (WHO) Policy Perspectives on Medicines. Pharmacovigilance: ensuring the safe use of medicines. 2004. http://apps.who.int/medicinedocs/pdf/s6164e/s6164e.pdf. Accessed 30 Aug 2017.

3. European Commission. Directive 2001/83/EC of the European Parliament and of the Council of 6 November 2001 on the Community code relating to medicinal products for human use. https ://ec.europa.eu/health/sites/health/files/files/eudralex/vol-1/ dir_2001_83_consol_2012/dir_2001_83_cons_2012_en.pdf. Accessed 31 Aug 2017.

4. European Commission. Regulation (EC) No 726/2004 of the European Parliament and of the Council of 31 March 2004 laying down Community procedures for the authorisation and supervision of medicinal products for human and veterinary use and establishing a European Medicines Agency. https://eur-lex.europ a.eu/legal-content/EN/TXT/PDF/?uri=CELEX:02004R0726 -20190128\&from=EN. Accessed 31 Aug 2017.

5. European Commission. Commission Implementing Regulation (EU) No 520/2012 of 19 June 2012 on the performance of pharmacovigilance activities provided for in Regulation (EC) No $726 / 2004$ of the European Parliament and of the Council and Directive 2001/83/EC of the European Parliament and of the Council. https://eur-lex.europa.eu/LexUriServ/LexUriServ.do?u ri=OJ:L:2012:159:0005:0025:EN:PDF. Accessed 31 Aug 2017.
6. European Medicines Agency (EMA) and Heads of Medicines Agencies. Good pharmacovigilance practices. http://www.ema. europa.eu/ema/index.jsp?curl=pages/regulation/document_listi ng/document_listing_000345.jsp. Accessed 30 Aug 2017.

7. European Medicines Agency (EMA) and Heads of Medicines Agencies. Guideline on good pharmacovigilance practices (GVP): Module VI-collection, management and submission of reports of suspected adverse reactions to medicinal products [EMA/873138/2011 Rev 2]. 2017. https://www.ema.europa.eu/ en/documents/regulatory-procedural-guideline/guideline-goodpharmacovigilance-practices-gvp-module-vi-collection-manag ement-submission-reports_en.pdf. Accessed 5 Aug 2017.

8. International Council for Harmonisation of Technical Requirements for Pharmaceuticals for Human Use (ICH) ICH E2D harmonised tripartite guideline: post-approval safety data management: definitions and standards for expedited reporting. 2003. http://www.ich.org/fileadmin/Public_Web_Site/ICH_Products/ Guidelines/Efficacy/E2D/Step4/E2D_Guideline.pdf. Accessed 12 Aug 2017.

9. European Medicines Agency (EMA) and Heads of Medicines Agencies. Guideline on good pharmacovigilance practices (GVP): Module VII—periodic safety update report [EMA/405655/2016]. 2013. https://www.ema.europa.eu/en/documents/scientific-guide line/guideline-good-pharmacovigilance-practices-gvp-modul e-vii-periodic-safety-update-report_en.pdf. Accessed 14 Aug 2017.

10. European Medicines Agency. Post-authorisation: regulatory and procedural guidance. https://www.ema.europa.eu/en/human-regul atory/post-authorisation. Accessed 14 Aug 2017.

11. European Medicines Agency (EMA) and Heads of Medicines Agencies. Guideline on good pharmacovigilance practices: Module V—risk management systems [MA/838713/2011]. 2017. https ://www.ema.europa.eu/en/documents/scientific-guideline/guide line-good-pharmacovigilance-practices-module-v-risk-manag ement-systems-rev-2_en.pdf. Accessed 31 Aug 2017.

12. European Medicines Agency (EMA) and Heads of Medicines Agencies. Guideline on good pharmacovigilance practices: Module IX — signal management [EMA/827661/2011]. 2017. https:// www.ema.europa.eu/en/documents/scientific-guideline/guidelinegood-pharmacovigilance-practices-gvp-module-ix-signal-manag ement-rev-1_en.pdf. Accessed 19 Aug 2017.

13. European Medicines Agency (EMA) and Heads of Medicines Agencies. Guideline on good pharmacovigilance practices (GVP) Module VIII - Post-authorisation safety studies [EMA/813938/2011 Rev 3*]. 2017. https://www.ema.europa.eu/ en/documents/scientific-guideline/guideline-good-pharmacovi gilance-practices-gvp-module-viii-post-authorisation-safety-studi es-rev-3_en.pdf. Accessed 31 Oct 2017.

14. Omar I, Harris E. The use of social media in ADR monitoring and reporting. J Pharmacovigil. 2016;4:223. https://doi. org/10.4172/2329-6887.1000223.

15. Toussi M, Chamberlain JL, Breckenridge A. Using social media as the patient's voice in the benefit-risk assessment of drugs: are we ready? Med Writ. 2015;24:2. https://doi.org/10.1179/20474 80615Z.000000000289.

16. Innovative Medicines Initiative (IMI). WEB-RADR: recognising adverse drug reactions. https://web-radr.eu/. Accessed $12 \mathrm{Sept}$ 2017.

17. Ghosh R, Lewis D. Aims and approaches of WEB-RADR: a consortium ensuring reliable ADR reporting via mobile devices and new insights from social media. Expert Opin Drug Saf. 2015;14(12):1845-53.

18. Ramble B, Balatero K. Deloitte Center for Health Solutions blog. Developing mission critical social listening programs for pharmaceutical companies. 2016. https://blogs.deloitte.com/centerforh 
ealthsolutions/developing-mission-critical-social-listening-progr ams-for-pharmaceutical-companies/. Accessed 19 July 2016.

19. Pal SN, Duncombe C, Falzon D, et al. WHO strategy for collecting safety data in public health programmes: complementing spontaneous reporting systems. Drug Saf. 2013;36(2):75-81. https ://doi.org/10.1007/s40264-012-0014-6.

20. Härmark L, van Grootheest AC. Pharmacovigilance: methods, recent developments and future perspectives. Eur J Clin Pharmacol. 2008;64(8):743-52. https://doi.org/10.1007/s0022 8-008-0475-9.

21. Freifeld CC, Brownstein JS, Menone CM, et al. Digital drug safety surveillance: monitoring pharmaceutical products in Twitter. Drug Saf. 2014;37(5):343-50. https://doi.org/10.1007/s4026 4-014-0155-x.

22. Duh MS, Ellman B, Van Audenrode M, Greenberg P, Cremieux P. Learning from social media for adverse event reporting. Law360, New York. 2017. https://www.analysisgroup.com/globalassets/ content/insights/publishing/learning_from_social_media.pdf. Accessed 31 Aug 2017.

23. International Council for Harmonisation of Technical Requirements for Pharmaceuticals for Human Use (ICH). https://www. ich.org/home.html. Accessed 13 Sept 2017.

24. International Council for Harmonisation of Technical Requirements for Pharmaceuticals for Human Use (ICH). Implementation guide for electronic transmission of individual case safety reports (ICSRs) E2B(R3) data elements and message specification [version 5.02]. 2016. http://estri.ich.org/e2br3/index.htm. Accessed 12 Sept 2017.

25. European Medicines Agency. EudraVigilance. https://www.ema. europa.eu/en/human-regulatory/research-development/pharm acovigilance/eudravigilance. Accessed 05 Feb 2019.

26. Lengsavath M, Dal Pra A, de Ferran A, Brosch S, Harmark L, Newbould V, et al. Social media monitoring and adverse drug reaction reporting in pharmacovigilance: an overview of the regulatory landscape. Ther Innov Regul Sci. 2016;51(1):12531. https://doi.org/10.1177/2168479016663264.

27. Brosch S, Newbould V. 1st workshop report: Innovative Medicines Initiative WEB-RADR workshop report: mobile technologies and social media as new tools in pharmacovigilance. IMI WEB-RADR. Report number: EMA/657669/2014, 2015. https ://webradr.files.wordpress.com/2015/09/1st-web-radr-works hop-report.pdf. Accessed 30 Aug 2017.

28. Brosch S, Newbould V. 2nd workshop report: Innovative Medicines Initiative WEB-RADR workshop report: mobile technologies and social media as new tools in pharmacovigilance. IMI WEB-RADR. Report number: EMA/70469/2017, 2017. http:// www.ema.europa.eu/docs/en_GB/document_library/Repor t/2017/02/WC500221615.pdf. Accessed 30 Aug 2017.

29. Maskell S, Sloane R, Perkins S, Heap J, Hajne J, Jones A, et al. Estimating the pertinent information present in social media, not just what an algorithm detects. Drug Saf. 2017;40:9371045. https://doi.org/10.1007/s40264-017-0580-8.

30. Caster O, Lerch M, Vroman B, van Stekelenborg J. Performance of disproportionality analysis for statistical signal detection in social media data [abstract]. Pharmacoepidemiol Drug Saf. 2016;25(S3):411.

31. Sloan R, Osanlou O, Lewis D, Bollegala D, Maskell S, Pirmohamed M. Social media and pharmacovigilance: a review of the opportunities and challenges. Br J Clin Pharmacol. 2015;80:910-20. https://doi.org/10.1111/bcp.12717.

32. Caster O, Dietrich J, Kürzinger ML, Lerch M, Maskell S, Norén GN, et al. Assessment of the utility of social media for broadranging statistical signal detection in pharmacovigilance: results from the WEB-RADR Project. Drug Saf. 2018;41(12):1355-69. https://doi.org/10.1007/s40264-018-0699-2.
33. Government Social Research (GSR). Social media research guidance. Using social media for social research: an introduction. https://assets.publishing.service.gov.uk/government/uploa ds/system/uploads/attachment_data/file/524750/GSR_Social_ Media_Research_Guidance_-_Using_social_media_for_socia 1_research.pdf. Accessed 28 Aug 2017.

34. European Commission. Digital single market: glossary. https ://ec.europa.eu/digital-single-market/en/glossary. Accessed 23 July 2017.

35. European Medicines Agency (EMA) and Heads of Medicines Agencies. Guideline on good pharmacovigilance practices (GVP) Annex I-definitions (Rev 4) [EMA/876333/2011 Rev 4]. 2017. http://www.ema.europa.eu/docs/en_GB/docum ent_library/Scientific_guideline/2013/05/WC500143294.pdf. Accessed 14 July 2017.

36. Association of British Pharmaceutical Industry (ABPI) Pharmacovigilance Expert Network. Guidance notes on the management of adverse events and product complaints from digital media. Version: 2.0. 2013. http://www.abpi.org.uk/media/1593/ abpi-guidance-on-pv-and-digital-media.pdf. Accessed 17 May 2017.

37. Banerjee AK, Ingate S. Web-based patient-reported outcomes in drug safety and risk management challenges and opportunities? Drug Saf. 2012;35(6):437-46.

38. Liang $\mathrm{H}, \mathrm{Fu} \mathrm{K}-\mathrm{W}$. Testing propositions derived from twitter studies: generalization and replication in computational social science. PLoS One. 2015;10(8):e0134270. https://doi.org/10.1371/journ al.pone. 0134270 .

39. Pimpalkhute P, Patki A, Nikfarjam A, Gonzalez G. Phonetic spelling filter for keyword selection in drug mention mining from social media. AMIA Summits Transl Sci Proc. 2014; 2014:90-5.

40. Paul JM, Sarker A, Brownstein JS, Nikfarjam A, Scotch M, Smith $\mathrm{KL}$, et al. Social media mining for public health monitoring and surveillance. Pac Symp Biocomput. 2016;21:468-79.

41. EUR-LEX. Regulation (EU) 2016/679 of the European Parliament and of the Council of 27 April 2016 on the protection of natural persons with regard to the processing of personal data and on the free movement of such data, and repealing Directive 95/46/ EC (General Data Protection Regulation). https://eur-lex.europ a.eu/legal-content/EN/TXT/?uri=celex:32016R0679. Accessed 10 Aug 2018.

42. Mittelstadt BD, Floridi L. The ethics of big data: current and foreseeable issues in biomedical contexts. Sci Eng Ethics. 2016;22(2):303-41. https://doi.org/10.1007/s11948-015-9652-2.

43. The European Network of Centres for Pharmacoepidemiology and Pharmacovigilance (ENCePP). Guide on methodological standards in pharmacoepidemiology (revision 3). http://www. encepp.eu/standards_and_guidances/methodologicalGuide.shtml. Accessed 3 May 2017.

44. European Medicines Agency. Guidance for the format and content of the protocol of non-interventional post-authorisation safety studies [EMA/623947/2012]. 2012. https://www.ema.europa.eu/ documents/other/guidance-format-content-protocol-non-interventi onal-post-authorisation-safety-studies_en.pdf. Accessed 3 May 2017.

45. Cowie JM, Gurney ME. The use of Facebook advertising to recruit healthy elderly people for a clinical trial: baseline metrics. JMIR Res Protoc. 2018;7(1):e20. https://doi.org/10.2196/resprot.7918.

46. Gelinas L, Pierce R, Winkler S, Glenn Cohen I, Lynch F, Bierer BE. Using social media as a research recruitment tool: ethical issues and recommendations. Am J Bioeth. 2017;17(3):3-14. https ://doi.org/10.1080/15265161.2016.1276644.

47. Banks L. Pharma gets social: 7 pharma social listening strategies. Pharmaphorum. 2017. https://pharmaphorum.com/views -and-analysis/pharma-gets-social-7-pharma-social-listening-strat egies/. Accessed 15 May 2017. 
48. Limaye N, Saraogi A. How social media is transforming pharma and healthcare. Appl Clin Trials. 2018;27(2). http://www.appli edclinicaltrialsonline.com/how-social-media-transforming-pharm a-and-healthcare. Accessed 11 Dec 2018.

49. Snelson CL. Qualitative and mixed methods social media research: a review of the literature. Int J Qual Methods. 2016;15(1):1-15. https://doi.org/10.1177/1609406915624574.

50. MS OnetoOne ${ }^{\circledR}$. Personalized multiple sclerosis support. https:// www.msonetoone.com/. Accessed 16 July 2017.

51. @Diabetes_Sanofi Twitter feed.https://twitter.com/diabetes_sanof i. Accessed 16 July 2017.

52. Househ M, Borycki E, Kushniruk A. Empowering patients through social media: the benefits and challenges. Health Inform J. 2014;20(1):50-8. https://doi.org/10.1177/1460458213476969.

53. Boucher JL. Technology and patient-provider interactions: improving quality of care, but is it improving communication and collaboration? Diabetes Spectr. 2010;23(3):142-4. https:// doi.org/10.2337/diaspect.23.3.142.

54. EMA Workshop on Patient Support and Market Research Programmes - spectrum of programmes falling under the terms of PSP and MRPs and the type of safety data collected. Pharmaceutical Industry Associations. http://www.ema.europa.eu/docs/ en_GB/document_library/Presentation/2013/06/WC50014466 7.pdf. Accessed 11 June 2017.

55. European Medicines Agency (EMA) and Heads of Medicines Agencies. Guideline on good pharmacovigilance practices (GVP): Module XVI-risk minimisation measures: selection of tools and effectiveness indicators [EMA/204715/2012 Rev 2]. 2017. https:// www.ema.europa.eu/en/documents/scientific-guideline/guidelinegood-pharmacovigilance-practices-module-xvi-risk-minimisati on-measures-selection-tools en-3.pdf. Accessed 14 July 2017.

56. Genzyme. LEMTRADA REMS (Risk Evaluation and Mitigation Strategy) program. https://www.lemtradarems.com/. Accessed 14 July 2017.

57. Sullivan HW, O'Donoghue AC, Amie C, Aikin KJ. Communicating benefit and risk information in direct-to-consumer print advertisements: a randomized study. Ther Innov Regul Sci. 2015;49(4):493-502.

58. Von Muhlen M, Ohno-Machado L. Reviewing social media use by clinicians. J Am Med Inform Assoc. 2012;19(5):777-81.

59. Topaz M, Lai K, Dhopeshwarkar N, Seger DL, Sa'adon R, Goss F, et al. Clinicians' reports in electronic health records versus patients' concerns in social media: a pilot study of adverse drug reactions of aspirin and atorvastatin. Drug Saf. 2016;39(3):241-50.

60. Bloom SB. Crossing the quality chasm: a new health system for the 21st century. JAMA. 2002;287(5):646-7. https://doi. org/10.1001/jama.287.5.646-JBK0206-3-1.
61. Rozenblum R, Bates DW. Patient-centred healthcare, social media and the internet: the perfect storm? BMJ Qual Saf. 2013;22(3):183-6.

62. Sarker A, Ginn R, Nikfarjam A, O'Connor K, Smith K, Jayaraman $\mathrm{S}$, et al. Utilizing social media data for pharmacovigilance: a review. J Biomed Inform. 2015;54:202-12.

63. Harpaz R, DuMouchel W, Shah NH, Madigan D, Ryan P, Friedman C. Novel data-mining methodologies for adverse drug event discovery and analysis. Clin Pharmacol Ther. 2012;91(3):1010-21.

64. Curtis JR, Chen L, Higginbotham P, Nowell WB, Gal-Levy R, Willig J, et al. Social media for arthritis-related comparative effectiveness and safety research and the impact of direct-toconsumer advertising. Arthritis Res Ther. 2017;19:48. https:// doi.org/10.1186/s13075-017-1251-y.

65. Kim K, Kwon N. Profile of e-patients: analysis of their cancer information-seeking from a national survey. J Health Commun. 2010;15(7):712-33.

66. Rajagopalan MS, Khanna VK, Leiter Y, Stott M, Showalter TN, Dicker AP, et al. Patient-oriented cancer information on the internet: a comparison of Wikipedia and a professionally maintained database. J Oncol Pract. 2011;7(5):319-23.

67. Nordqvist C, Hanberger L, Timpka T, Nordfeldt S. Health professionals' attitudes towards using a Web 2.0 portal for child and adolescent diabetes care: qualitative study. J Med Internet Res. 2009;11(2):e12.

68. Greene JA, Choudhry NK, Kilabuk E, Shrank WH. Online social networking by patients with diabetes: a qualitative evaluation of communication with Facebook. J Gen Intern Med. 2011;26(3):287-92.

69. Sanford A. "I can air my feelings instead of eating them": blogging as social support for the morbidly obese. Commun Stud. 2010;61(5):567-84.

70. Council for International Organizations of Medical Sciences (CIOMS). Report of CIOMS Working Group VIII on Practical Aspects of Signal Detection in Pharmacovigilance. Geneva: CIOMS; 2010.

71. Pierce CE, Bouri K, Pamer C, Proestel S, Rodriguez HW, Van Le H, et al. Evaluation of Facebook and Twitter monitoring to detect safety signals for medical products: an analysis of recent FDA safety alerts. Drug Saf. 2017;40(4):317-31. https://doi. org/10.1007/s40264-016-0491-0.

72. Dunn AG, Leask J, Zhou X, Mandl KD, Coiera E. Associations between exposure to and expression of negative opinions about human papillomavirus vaccines on social media: an observational study. J Med Internet Res. 2015;17(6):e144. https://doi. org/10.2196/jmir.4343. 